\title{
Varied Immune Response to FVIII: Presence of Proteolytic Antibodies Directed to Factor VIII in Different Human Pathologies
}

\author{
Bharath Wootla $\cdot$ Desirazu Narasimha Rao • \\ Alain Friboulet • Taizo Uda • \\ Sébastien Lacroix-Desmazes • Srini V. Kaveri
}

Published online: 20 May 2009

(C) Humana Press Inc. 2009

\section{Erratum to: Clinic Rev Allerg Immunol DOI 10.1007/s12016-009-8116-3}

In the original version of this article, one of the author names was presented incorrectly. "Narasimha Rao Desirazu" should read "Desirazu Narasimha Rao".

The online version of the original article can be found under doi:10.1007/s12016-009-8116-3.

B. Wootla $\cdot$ S. Lacroix-Desmazes $\cdot$ S. V. Kaveri

A. Friboulet

Université de Technologie de Compiègne,

Centre de Recherche des Cordeliers,

Université Pierre et Marie Curie,

Paris6, UMR S 872,

Paris F-75006, France

BP 2052960205 ,

Compiègne Cedex, France

B. Wootla $\cdot$ S. Lacroix-Desmazes $\cdot$ S. V. Kaveri

Université Paris Descartes,

UMR S 872,

Paris F-75006, France

B. Wootla $\cdot$ S. Lacroix-Desmazes $\cdot$ S. V. Kaveri

INSERM,

U872,

Paris F-75006, France

T. Uda

Research Center for Applied Medical Engineering,

Oita University,

Dan-noharu 700, Oita-shi,

Oita 870-1192, Japan

D. N. Rao

Department of Biochemistry, Indian Institute of Science,

Bangalore, India

\author{
S. Lacroix-Desmazes $(\square)$ \\ Centre de Recherche des Cordeliers, INSERM, \\ UMR S 872 Equipe 16, 15 rue de l'école de Medicine, \\ 75006 Paris, France \\ e-mail: Sebastien.Lacroix-Desmazes@crc.jussieu.fr
}

